\title{
Experimental and Theoretical Studies on the Tautomerism in 2-Aminopyridines and 2(1H)-Pyridinones: Synthesis of 2-Amino-4-aryl-3-cyano-6-(3,4-dimethoxyphenyl)py- ridines and 4-Aryl-3-cyano-6-(3,4-dimethoxyphenyl)-2(1H)-pyridinones
}

\author{
Abolghasem Davoodnia, ${ }^{*}$ Paria Attar, Ali Morsali, Hossein Eshghi, ${ }^{\dagger}$ Niloofar Tavakoli-Hoseini, and Shahriar Khadem ${ }^{\ddagger}$ \\ Department of Chemistry, Mashhad Branch, Islamic Azad University, Mashhad, Iran. *E-mail: adavoodnia@mshdiau.ac.ir \\ ${ }^{\dagger}$ Department of Chemistry, Faculty of Sciences, Ferdowsi University of Mashhad, Mashhad, Iran \\ ¿Department of Chemistry, University of Ottawa, Ottawa, Ontario, Canada \\ Received March 24, 2011, Accepted April 7, 2011
}

\begin{abstract}
Under solvent-free conditions and in one-pot, a series of 2-amino-4-aryl-3-cyano-6-(3,4-dimethoxyphenyl)pyridines and 4-aryl-3-cyano-6-(3,4-dimethoxyphenyl)-2(1H)-pyridinones were prepared using 3,4dimethoxyacetophenone, an aldehyde, malononitrile (or ethyl cyanoacetate), and ammonium acetate in the

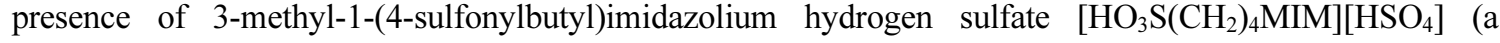
Brønsted acidic ionic liquid) as the catalyst in very short reaction time. The preference for the formation of more stable tautomers was consistence with the theoretical calculation using the Gaussian 03 program at the B3LYP hybrid density functional level.
\end{abstract}

Key Words : Brønsted-acidic ionic liquids, 3-Cyanopyridines, 3-Cyanopyridinones, Multicomponent reactions, Tautomerism

\section{Introduction}

Pyridine derivatives have occupied an unique position in medicinal chemistry. Besides many naturally occurring pyridines, several synthetic derivatives show interesting biological activities. For examples, 2-amino-3-cyanopyridines have antibacterial, ${ }^{1}$ antimicrobial, ${ }^{2,3}$ antifungal, ${ }^{4}$ and cardiotonic $^{5}$ activities. They are also found to be selective IKK- $\beta$ serine-threonine protein kinase inhibitors. ${ }^{6}$ On the other hand, 3-cyanopyridinone derivatives have antineoplastic, ${ }^{7}$ PDE3 inhibitory, ${ }^{8}$ cardiotonic, ${ }^{5,9}$ and colon tumor cell growth inhibitory ${ }^{10}$ effects. During past three decades, many synthetic methods have been used for the preparation of these 3-cyanopyridine, ${ }^{1-6,11-14}$ and 3-cyanopyridinone derivatives. ${ }^{5,7,9,10,15-18}$ As part of our efforts towards the development of synthetic methodologies, ${ }^{19-27}$ here we report our investigation on the application of 3-methyl-1-(4-sulfonylbutyl)imidazolium hydrogen sulfate $\left[\mathrm{HO}_{3} \mathrm{~S}\left(\mathrm{CH}_{2}\right)_{4} \mathrm{MIM}\right]-$ $\left[\mathrm{HSO}_{4}\right]$, a Brønsted acidic ionic liquid as a reusable catalyst, ${ }^{28}$ for the synthesis of 3-cyanopyridines and 3-cyanopyridinones in high yields. For these syntheses, a mixture of 3,4-dimethoxyacetophenone, different aldehydes, malononitrile (or ethyl cyanoacetate), and ammonium acetate (a multicomponent reaction) was used in solvent-free conditions. Moreover, theoretical calculations were performed at the B3LYP hybrid density functional level using the Gaussian 03 program to predict the more stable tautomers. Geometries of tautomers were optimized at $6-31+G(d, p)$ basis sets. The calculated frequencies have no imaginary vibrational frequency, indicating that the optimized geometries are reasonable and reliable. These results are also presented here.

\section{Experimental}

All chemicals were commercially available and used as such. The Brønsted-acidic ionic liquid $\left[\mathrm{HO}_{3} \mathrm{~S}\left(\mathrm{CH}_{2}\right)_{4} \mathrm{MIM}\right]$ $\left[\mathrm{HSO}_{4}\right]$ was prepared using a literature method. ${ }^{28}$ Melting points were recorded on an electrothermal type 9100 melting point apparatus. The IR spectra were obtained on a Shimadzu spectrophotometer as $\mathrm{KBr}$ disks. The ${ }^{1} \mathrm{H}-\mathrm{NMR}(500 \mathrm{MHz})$ and ${ }^{13} \mathrm{C}-\mathrm{NMR}$ spectra $(125 \mathrm{MHz})$ were recorded on a Bruker DRX500 spectrometer. Mass spectrum was recorded on a Finnigan-MAT 8430 mass spectrometer operating at an ionization potential of $70 \mathrm{eV}$.

General Procedure for the Synthesis of 2-Amino-4aryl-3-cyano-6-(3,4-dimethoxyphenyl)pyridines B1-B5 and 4-Aryl (or alkyl)-3-cyano-6-(3,4-dimethoxyphenyl)2(1H)-pyridinones D1-D5. A mixture of 3,4-dimethoxyacetophenone $(2 \mathrm{mmol})$, an aldehyde $(2 \mathrm{mmol})$, malononitrile (or ethyl cyanoacetate) $(2 \mathrm{mmol})$, ammonium acetate (16 mmol), and $\left[\mathrm{HO}_{3} \mathrm{~S}\left(\mathrm{CH}_{2}\right)_{4} \mathrm{MIM}\right]\left[\mathrm{HSO}_{4}\right](10 \mathrm{~mol} \%$ based on the aldehyde) was heated on the oil bath at $150{ }^{\circ} \mathrm{C}$ for a few minutes. After this time, the reaction mixture was cooled to room temperature, and cold ethanol was added. The crude product was collected and recrystallized from ethanol to give compounds B1-B5 and D1-D5 in high yields.

Computational Methodology. All calculations were performed at the B3LYP hybrid density functional level using the Gaussian 03 program. Geometries of tautomers were optimized at $6-31+G(d, p)$ basis sets. The calculation of quantum chemical energies is described as follows: all molecules were preoptimized using AM1 semiempirical method implemented in the Chemoffice 2004 program. The final geometries were obtained at B3LYP/6-31+G(d,p) level 
using OPT keyword and then frequency calculations were done using FREQ keyword. Zero point energy corrections and thermal free energies have been obtained from frequency calculations. The total energy of molecule is the sum of electronic and zero point energy. All geometries have no imaginary vibrational frequency, indicating that they should be stable.

\section{Spectral Data for New Compounds.}

2-Amino-3-cyano-6-(3,4-dimethoxyphenyl)-4-phenylpyridine (B1): ${ }^{1} \mathrm{H} \mathrm{NMR}\left(500 \mathrm{MHz}, \mathrm{CDCl}_{3}, \delta \mathrm{ppm}\right): 3.98$ (s, $\left.3 \mathrm{H}, \mathrm{OCH}_{3}\right), 4.03\left(\mathrm{~s}, 3 \mathrm{H}, \mathrm{OCH}_{3}\right), 5.37$ (s, 2H, NH 2$), 6.98$ (d, $1 \mathrm{H}, J=8.5 \mathrm{~Hz}$, arom-H), $7.20(\mathrm{~s}, 1 \mathrm{H}$, arom-H), 7.50-7.70 $\left(\mathrm{m}, 7 \mathrm{H}\right.$, arom-H); ${ }^{13} \mathrm{C} \mathrm{NMR}\left(\mathrm{CDCl}_{3} \&\right.$ DMSO- $\left.d_{6}, \delta \mathrm{ppm}\right)$ : 56.41, 56.46, 87.95, 110.68, 110.78, 111.40, 117.57, 120.86, $127.57,128.54,129.29,130.17,137.43,149.61,151.50$, 155.49, 159.34, 160.53; IR ( $\mathrm{KBr}$ disc): $v$ 3530, $3406\left(\mathrm{NH}_{2}\right)$, $2200(\mathrm{CN}) \mathrm{cm}^{-1}$; MS, $\mathrm{m} / z 331\left(M^{+}\right)$.

2-Amino-4-(4-bromophenyl)-3-cyano-6-(3,4-dimethoxyphenyl)pyridine (B2). ${ }^{1} \mathrm{H} \mathrm{NMR}\left(500 \mathrm{MHz}, \mathrm{CDCl}_{3}, \delta \mathrm{ppm}\right)$ : $3.85\left(\mathrm{~s}, 3 \mathrm{H}, \mathrm{OCH}_{3}\right), 3.89\left(\mathrm{~s}, 3 \mathrm{H}, \mathrm{OCH}_{3}\right), 5.55\left(\mathrm{~s}, 2 \mathrm{H}, \mathrm{NH}_{2}\right)$, $6.86(\mathrm{~d}, 1 \mathrm{H}, J=8.4 \mathrm{~Hz}$, arom-H), $7.01(\mathrm{~s}, 1 \mathrm{H}$, arom-H), 7.40-7.60 (m, $6 \mathrm{H}$, arom-H); ${ }^{13} \mathrm{C} \mathrm{NMR}\left(\mathrm{CDCl}_{3} \& \mathrm{DMSO}-d_{6}\right.$, dppm): 56.36, 56.39, 87.30, 110.27, 110.67, 111.37, 117.49, 120.82 , 124.46, 130.15, 130.88, 132.43, 136.41, 149.53, $151.45,153.92,159.79,160.73$; IR (KBr disc): $v$ 3487, 3350 $\left(\mathrm{NH}_{2}\right), 2217(\mathrm{CN}) \mathrm{cm}^{-1}$; MS, $m / z 409\left(M^{+}\right), 411\left(M^{+}+2\right)$.

2-Amino-4-(4-chlorophenyl)-3-cyano-6-(3,4-dimethoxyphenyl)pyridine (B3). ${ }^{1} \mathrm{H}$ NMR $\left(500 \mathrm{MHz}, \mathrm{DMSO}-d_{6}\right.$, Sppm): $3.82\left(\mathrm{~s}, 3 \mathrm{H}, \mathrm{OCH}_{3}\right), 3.84\left(\mathrm{~s}, 3 \mathrm{H}, \mathrm{OCH}_{3}\right), 6.96(\mathrm{~s}, 2 \mathrm{H}$, $\left.\mathrm{NH}_{2}\right), 7.05(\mathrm{~d}, 1 \mathrm{H}, J=8.5 \mathrm{~Hz}$, arom-H), 7.27 (s, 1H, arom$\mathrm{H}), 7.60-7.80$ (m, 6H, arom-H); ${ }^{13} \mathrm{C}$ NMR (DMSO- $\left.d_{6}, \delta p p m\right)$ : 56.45, 56.52, 86.48, 109.47, 111.44, 112.34, 117.92, 121.43, $129.59,130.88,131.14,135.26,136.83,149.62,151.69$, 154.24, 159.39, 161.54; IR ( $\mathrm{KBr}$ disc): $v$ 3490, $3349\left(\mathrm{NH}_{2}\right)$, $2217(\mathrm{CN}) \mathrm{cm}^{-1}$; MS, $m / z 365\left(M^{+}\right), 367\left(M^{+}+2\right)$.

2-Amino-3-cyano-6-(3,4-dimethoxyphenyl)-4-(4-hydroxyphenyl)pyridine (B4). ${ }^{1} \mathrm{H}$ NMR $\left(500 \mathrm{MHz}, \mathrm{DMSO}-d_{6}\right.$, Sppm): $3.81\left(\mathrm{~s}, 3 \mathrm{H}, \mathrm{OCH}_{3}\right), 3.84\left(\mathrm{~s}, 3 \mathrm{H}, \mathrm{OCH}_{3}\right), 6.81(\mathrm{~s}, 2 \mathrm{H}$, $\left.\mathrm{NH}_{2}\right), 6.91$ (d, 2H, $J=7.7 \mathrm{~Hz}$, arom-H), 7.04 (d, 1H, $J=8.5$ $\mathrm{Hz}$, arom-H), 7.20 (s, 1H, arom-H), 7.53 (d, 2H, $J=7.7 \mathrm{~Hz}$, arom-H), 7.69 (s, 1H, arom-H), $7.72(\mathrm{~d}, 1 \mathrm{H}, J=8.2 \mathrm{~Hz}$, arom-H), 9.90 (s br., $1 \mathrm{H}, \mathrm{OH}) ;{ }^{13} \mathrm{C}$ NMR (DMSO- $d_{6}, \delta \mathrm{ppm}$ ): 56.45, 56.51, 86.34, 109.25, 111.40, 112.34, 116.31, 118.47, $121.27,128.49,130.71,131.16,149.58,151.51,155.42$, 158.99, 159.70, 161.70; IR ( $\mathrm{KBr}$ disc): v 3424, $3335\left(\mathrm{NH}_{2}\right)$, $2231(\mathrm{CN}) \mathrm{cm}^{-1}$; MS, $\mathrm{m} / z 347\left(M^{+}\right)$.

2-Amino-3-cyano-6-(3,4-dimethoxyphenyl)-4-(4-methoxyphenyl)pyridine (B5). ${ }^{1} \mathrm{H}$ NMR $\left(500 \mathrm{MHz}, \mathrm{CDCl}_{3}\right.$, Sppm): 3.92 (s, 3H, $\left.\mathrm{OCH}_{3}\right), 3.98\left(\mathrm{~s}, 3 \mathrm{H}, \mathrm{OCH}_{3}\right), 4.03(\mathrm{~s}, 3 \mathrm{H}$, $\left.\mathrm{OCH}_{3}\right), 5.40\left(\mathrm{~s}, 2 \mathrm{H}, \mathrm{NH}_{2}\right), 6.98(\mathrm{~d}, 1 \mathrm{H}, J=8.4 \mathrm{~Hz}$, arom-H), $7.08(\mathrm{~d}, 2 \mathrm{H}, J=8.7 \mathrm{~Hz}$, arom-H), 7.17 (s, 1H, arom-H), 7.60 $\left(\mathrm{dd}, 1 \mathrm{H},{ }^{3} J_{1}=8.4 \mathrm{~Hz},{ }^{4} J_{2}=1.9 \mathrm{~Hz}\right.$, arom-H), $7.64(\mathrm{~d}, 2 \mathrm{H}, J=$ $8.7 \mathrm{~Hz}$, arom-H), $7.67\left(\mathrm{~d}, 1 \mathrm{H},{ }^{4} \mathrm{~J}=1.9 \mathrm{~Hz}\right.$, arom-H); ${ }^{13} \mathrm{C}$ NMR $\left(\mathrm{CDCl}_{3}, \delta \mathrm{ppm}\right): 55.86,56.44,56.47,87.78,110.67$, $110.78,111.41,114.80,117.98,120.77,129.72,130.03$, $131.12,149.67,151.45,155.01,159.49,160.63,161.39$; IR (KBr disc): v 3494, $3348\left(\mathrm{NH}_{2}\right), 2214(\mathrm{CN}) \mathrm{cm}^{-1}$; MS, $\mathrm{m} / z$

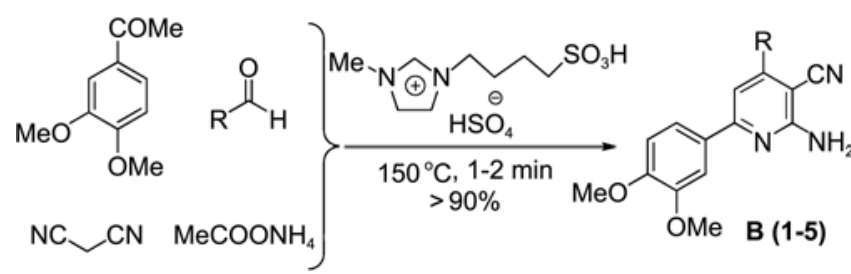

Scheme 1. Synthesis of 2-amino-4-aryl-3-cyano-6-(3,4-dimethoxyphenyl)pyridines.

$361\left(M^{+}\right)$

\section{Results and Discussion}

2-Amino-4-aryl-3-cyano-6-(3,4-dimethoxyphenyl)pyridines were synthesized using the known $\operatorname{method}^{11}$ (Scheme 1). The optimum conditions for using the catalyst $\left[\mathrm{HO}_{3} \mathrm{~S}_{\left(\mathrm{CH}_{2}\right)_{4}-}\right.$ $\mathrm{MIM}]\left[\mathrm{HSO}_{4}\right]$, temperature, solvent, and time were investigated. A model compound (4-chlorophenyl derivative) was prepared using different conditions. As shown in Table 1 and 2 , the best result was obtained in a solvent-free condition and $150{ }^{\circ} \mathrm{C}$ using $10 \mathrm{~mol} \%$ of catalyst. Several compounds were synthesized using the optimized conditions. The results were presented in Table 3. In all cases, tautomers $\mathbf{B}$ were the only observed products.

In a similar manner, several 4-aryl(or alkyl)-3-cyano-6(3,4-dimethoxyphenyl)-2(1H)-pyridinones are also prepar-

Table 1. Effect of the amounts of $\left[\mathrm{HO}_{3} \mathrm{~S}\left(\mathrm{CH}_{2}\right)_{4} \mathrm{MIM}\right]\left[\mathrm{HSO}_{4}\right]$ on the model reaction ${ }^{a}$

\begin{tabular}{cccc}
\hline Entry & Catalyst $(\mathrm{mol} \%)$ & Time $(\mathrm{min})^{\circ}$ & Yield $^{b}(\%)$ \\
\hline 1 & None & 5 & trace \\
2 & 5 & 2 & 81 \\
3 & 10 & 1.5 & 92 \\
4 & 15 & 1.5 & 91 \\
5 & 20 & 1.5 & 91 \\
6 & 25 & 1.5 & 92 \\
\hline
\end{tabular}

$a_{2}$ mmol 3,4-dimethoxyacetophenone, $2 \mathrm{mmol}$ 4-chlorobenzaldehyde, 2 $\mathrm{mmol}$ malononitrile, and $16 \mathrm{mmol}$ ammonium acetate under solvent-free conditions at $150{ }^{\circ} \mathrm{C}$. ${ }^{b}$ The yields were calculated based on 4-chlorobenzaldehyde and refer to the pure isolated product.

Table 2. Synthesis of 2-amino-4-chlorophenyl-3-cyano-6-(3,4-dimethoxyphenyl)pyridine $\mathbf{B 3}$ in the presence of $\left[\mathrm{HO}_{3} \mathrm{~S}\left(\mathrm{CH}_{2}\right)_{4} \mathrm{MIM}\right]-$ $\left[\mathrm{HSO}_{4}\right](10 \mathrm{~mol} \%)$ at different temperatures in solvent-free conditions $^{a}$

\begin{tabular}{cccc}
\hline Entry & Temperature $\left({ }^{\circ} \mathrm{C}\right)$ & Time $(\mathrm{min})$ & Yield $^{b}(\%)$ \\
\hline 1 & 120 & 5 & 83 \\
2 & 150 & 1.5 & 92 \\
3 & 150 & 5 & 92 \\
4 & 170 & 1.5 & 91 \\
5 & 190 & 1.5 & 92 \\
\hline
\end{tabular}

$\overline{a_{2} \text { mmol 3,4-dimethoxyacetophenone, } 2 \mathrm{mmol} \text { 4-chlorobenzaldehyde, } 2}$ mmol malononitrile, and $16 \mathrm{mmol}$ ammonium acetate. ${ }^{b}$ The yields were calculated based on 4-chlorobenzaldehyde and refer to the pure isolated product. 
Table 3. Synthesis of 2-amino-4-aryl-3-cyano-6-(3,4-dimethoxyphenyl)pyridines B1-B5 and 4-aryl(alkyl)-3-cyano-6-(3,4-dimethoxyphenyl)-2(1H)-pyridinones D1-D5 ${ }^{a}$

\begin{tabular}{cllcccc}
\hline \multirow{2}{*}{ Entry } & \multicolumn{1}{c}{$\mathrm{R}$} & \multirow{2}{*}{ Products } & \multirow{2}{*}{$\begin{array}{c}\text { Time } \\
(\mathrm{min})\end{array}$} & $\begin{array}{c}\text { Yields } \\
(\%)^{b}\end{array}$ & \multicolumn{2}{c}{$\mathrm{mp}\left({ }^{\circ} \mathrm{C}\right)$} \\
\hline 1 & $\mathrm{C}_{6} \mathrm{H}_{5}$ & $\mathbf{B 1}$ & 2 & 90 & $218-220$ & - \\
2 & $4-\mathrm{BrC}_{6} \mathrm{H}_{4}$ & $\mathbf{B 2}$ & 1 & 97 & $220-222$ & - \\
3 & $4-\mathrm{ClC}_{6} \mathrm{H}_{4}$ & $\mathbf{B 3}$ & 1.5 & 92 & $210-211$ & - \\
4 & $4-\mathrm{HOC}_{6} \mathrm{H}_{4}$ & B4 & 1.5 & 91 & $190-192$ & - \\
5 & $4-\mathrm{CH}_{3} \mathrm{OC}_{6} \mathrm{H}_{4}$ & B5 & 1.5 & 97 & $175-178$ & - \\
6 & $\mathrm{C}_{6} \mathrm{H}_{5}$ & D1 & 1.5 & 92 & $290-291$ & $285^{17}$ \\
7 & $4-\mathrm{ClC}_{6} \mathrm{H}_{4}$ & D2 & $<1$ & 98 & $291-293$ & $295^{17}$ \\
8 & $4-\mathrm{HOC}_{6} \mathrm{H}_{4}$ & D3 & $<1$ & 98 & $298-299$ & $311^{17}$ \\
9 & $4-\mathrm{CH}_{3} \mathrm{OC}_{6} \mathrm{H}_{4}$ & D4 & $<1$ & 97 & $262-264$ & $266^{17}$ \\
10 & $\mathrm{CH}_{3}$ & D5 & 1.5 & 90 & $260-262$ & $255^{17}$ \\
\hline
\end{tabular}

$a_{2}$ mmol 3,4-dimethoxyacetophenone, $2 \mathrm{mmol}$ aryl (or alkyl) aldehyde, 2 mmol malononitrile (or ethyl cyanoacetate), and $16 \mathrm{mmol}$ ammonium acetate in the presence of $\left[\mathrm{HO}_{3} \mathrm{~S}\left(\mathrm{CH}_{2}\right)_{4} \mathrm{MIM}\right]\left[\mathrm{HSO}_{4}\right](10 \mathrm{~mol} \%$ based on aldehyde) under solvent-free conditions at $150{ }^{\circ} \mathrm{C}$. ${ }^{b}$ The yields were calculated based on aldehyde and refer to the pure isolated product.

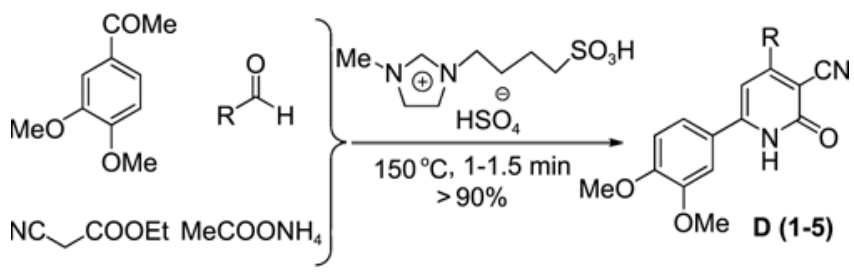

Scheme 2. Synthesis of 4-aryl (or alkyl)-3-cyano-6-(3,4-dimethoxyphenyl)-2(1H)-pyridinones.<smiles>[R]c1cc(-c2ccc(OC)c(OC)c2)[nH]c(=N)c1C#N</smiles><smiles>[R]c1cc(-c2ccc(OC)c(OC)c2)nc(O)c1C#N</smiles>

Scheme 3. Tautomers which have not formed in these syntheses.
Table 4. $\Delta \mathrm{E}, \Delta \mathrm{G}$ (in $\mathrm{kcal} / \mathrm{mol}$ ) and equilibrium constants at B3LYP using the $6-31 \mathrm{G}(\mathrm{d}, \mathrm{p})$ basis set

\begin{tabular}{lcrcr}
\hline $\mathrm{R}$ & $\Delta \mathrm{E}$ & $\mathrm{B} 3 L Y P$ & \multicolumn{1}{c}{$\Delta \mathrm{G}$} & B3LYP \\
\hline $\mathrm{C}_{6} \mathrm{H}_{5}$ & $\mathrm{E}_{\mathrm{A} 1}-\mathrm{E}_{\mathrm{B} 1}$ & 16.12 & $\mathrm{G}_{\mathrm{A} 1}-\mathrm{G}_{\mathrm{B} 1}$ & 16.24 \\
$4-\mathrm{BrC}_{6} \mathrm{H}_{4}$ & $\mathrm{E}_{\mathrm{A} 2}-\mathrm{E}_{\mathrm{B} 2}$ & 15.28 & $\mathrm{G}_{\mathrm{A} 2}-\mathrm{G}_{\mathrm{B} 2}$ & 16.45 \\
$4-\mathrm{ClC}_{6} \mathrm{H}_{4}$ & $\mathrm{E}_{\mathrm{A} 3}-\mathrm{E}_{\mathrm{B} 3}$ & 17.80 & $\mathrm{G}_{\mathrm{A} 3}-\mathrm{G}_{\mathrm{B} 3}$ & 17.60 \\
$4-\mathrm{HOC}_{6} \mathrm{H}_{4}$ & $\mathrm{E}_{\mathrm{A} 4}-\mathrm{E}_{\mathrm{B} 4}$ & 16.12 & $\mathrm{G}_{\mathrm{A} 4}-\mathrm{G}_{\mathrm{B} 4}$ & 16.24 \\
$4-\mathrm{CH}_{3} \mathrm{OC}_{6} \mathrm{H}_{4}$ & $\mathrm{E}_{\mathrm{A} 5}-\mathrm{E}_{\mathrm{B} 5}$ & 17.57 & $\mathrm{G}_{\mathrm{A} 5}-\mathrm{G}_{\mathrm{B} 5}$ & 17.45 \\
$\mathrm{C}_{6} \mathrm{H}_{5}$ & $\mathrm{E}_{\mathrm{C} 1}-\mathrm{E}_{\mathrm{D} 1}$ & 2.40 & $\mathrm{G}_{\mathrm{C1}}-\mathrm{G}_{\mathrm{D} 1}$ & 1.26 \\
$4-\mathrm{ClC}_{6} \mathrm{H}_{4}$ & $\mathrm{E}_{\mathrm{C} 2}-\mathrm{E}_{\mathrm{D} 2}$ & 2.45 & $\mathrm{G}_{\mathrm{C} 2}-\mathrm{G}_{\mathrm{D} 2}$ & 2.38 \\
$4-\mathrm{HOC}_{6} \mathrm{H}_{4}$ & $\mathrm{E}_{\mathrm{C} 3}-\mathrm{E}_{\mathrm{D} 3}$ & 2.54 & $\mathrm{G}_{\mathrm{C} 3}-\mathrm{G}_{\mathrm{D} 3}$ & 2.24 \\
$4-\mathrm{CH}_{3} \mathrm{OC}_{6} \mathrm{H}_{4}$ & $\mathrm{E}_{\mathrm{C} 4}-\mathrm{E}_{\mathrm{D} 4}$ & 2.51 & $\mathrm{G}_{\mathrm{C} 4}-\mathrm{G}_{\mathrm{D} 4}$ & 2.15 \\
$\mathrm{CH}_{3}$ & $\mathrm{E}_{\mathrm{C} 5}-\mathrm{E}_{\mathrm{D} 5}$ & 2.28 & $\mathrm{G}_{\mathrm{C} 5}-\mathrm{G}_{\mathrm{D} 5}$ & 2.48 \\
\hline
\end{tabular}<smiles>[R]c1cc(C#N)c(=NN)[nH]c1[R]</smiles>

A Tautomers<smiles>[R]c1nc(OO)c(C#N)c([R])c1[2H]</smiles>

C Tautomers<smiles>[R]c1nc(NP)c(C#N)c([R])c1[R]</smiles>

B Tautomers<smiles>[R]c1[nH]c(=O)c(C#N)c([R])c1[R]</smiles>

D Tautomers
Figure 1. Structure of Tautomers A-D.

ed. In these cases, however, ethyl cyanoacetate was used instead of malononitrile (Scheme 2) and tautomers $\mathbf{D}$ are the predominant products (Table 3 ).

To compare the stability of the tautomers, theoretical calculations have been performed on each pair of compounds. These calculations showed that tautomers $\mathbf{B}$ and $\mathbf{D}$ are more stable than A and $\mathbf{C}$, respectively (see Scheme 3).

Table 5. Some of the bond lengths (in $\AA$ ) for $\mathbf{B}$ and A tautomers

\begin{tabular}{l|lcccc|ccccc}
\hline \multirow{2}{*}{ Bond } & \multicolumn{3}{|c}{ B Tautomer } & \multicolumn{4}{c}{ A Tautomer } \\
\cline { 2 - 11 } & \multicolumn{1}{|c}{ B1 } & B2 & B3 & B4 & B5 & A1 & A2 & A3 & A4 & A5 \\
\hline r(N1)-r(H) & & & & & & 1.0133 & 1.01339 & 1.01335 & 1.01312 & 1.01309 \\
r(N1)-r(C2) & 1.33743 & 1.33775 & 1.33698 & 1.33703 & 1.33642 & 1.40520 & 1.40543 & 1.40546 & 1.40477 & 1.40466 \\
r(C2)-r(N3) & 1.36295 & 1.36026 & 1.36534 & 1.36314 & 1.36733 & 1.28586 & 1.28541 & 1.28542 & 1.28625 & 1.28643 \\
r(N3)-r(H) & 1.00793 & 1.00740 & 1.00831 & 1.00785 & 1.00847 & 1.01868 & 1.01868 & 1.01868 & 1.01867 & 1.01867 \\
r(N3)-r(H) & 1.0081 & 1.00755 & 1.00881 & 1.008 & 1.00899 & & & & \\
r(C2)-r(C4) & 1.42868 & 1.42908 & 1.42766 & 1.42868 & 1.42751 & 1.47192 & 1.47179 & 1.47182 & 1.47141 & 1.47137 \\
r(C4)-r(C5) & 1.4245 & 1.42402 & 1.42419 & 1.42415 & 1.42429 & 1.42464 & 1.42437 & 1.42437 & 1.42421 & 1.42423 \\
r(C5)-r(N6) & 1.1668 & 1.16685 & 1.16684 & 1.16703 & 1.16706 & 1.16623 & 1.16619 & 1.16623 & 1.16649 & 1.16652 \\
r(C4)-r(C7) & 1.4116 & 1.41121 & 1.41221 & 1.41285 & 1.4135 & 1.39082 & 1.39045 & 1.39094 & 1.39268 & 1.39292 \\
r(C7)-r(R) & 1.48671 & 1.48574 & 1.48581 & 1.48359 & 1.48261 & 1.48745 & 1.48642 & 1.48686 & 1.48362 & 1.48298 \\
r(C7)-r(C8) & 1.39730 & 1.39661 & 1.39633 & 1.39851 & 1.39832 & 1.42558 & 1.42495 & 1.42521 & 1.42689 & 1.4273 \\
r(C8)-r(H) & 1.08204 & 1.08196 & 1.08192 & 1.08193 & 1.08532 & 1.08098 & 1.08109 & 1.08105 & 1.08094 & 1.08097 \\
r(C8)-r(C9) & 1.40487 & 1.40559 & 1.40626 & 1.40416 & 1.40444 & 1.37799 & 1.37857 & 1.37865 & 1.37746 & 1.37712 \\
r(C9)-r(R') & 1.48586 & 1.48571 & 1.48477 & 1.48637 & 1.48558 & 1.47844 & 1.47813 & 1.47827 & 1.47876 & 1.47893 \\
r(C9)-r(N1) & 1.34337 & 1.34316 & 1.34457 & 1.34393 & 1.34548 & 1.36098 & 1.36061 & 1.36053 & 1.36111 & 1.3613 \\
\hline
\end{tabular}


Table 6. Some of the dihedral angels (in degrees) for $\mathbf{B}$ and $\mathbf{A}$ tautomers

\begin{tabular}{|c|c|c|c|c|c|c|c|c|c|c|}
\hline \multirow{2}{*}{ Angel } & \multicolumn{5}{|c|}{ B Tautomer } & \multicolumn{5}{|c|}{ A Tautomer } \\
\hline & $\mathrm{B} 1$ & B2 & B3 & B4 & B5 & A1 & $\mathrm{A} 2$ & A3 & A4 & A5 \\
\hline$<(\mathrm{H}-\mathrm{N} 1-\mathrm{C} 2)$ & & & & & & 113.5571 & 113.564 & 113.544 & 113.6002 & 113.6169 \\
\hline$<(\mathrm{N} 1-\mathrm{C} 2-\mathrm{N} 3)$ & 116.4884 & 116.5608 & 116.5442 & 116.4758 & 116.4803 & 116.4891 & 116.5216 & 116.5077 & 116.4445 & 116.4431 \\
\hline$<(\mathrm{H}-\mathrm{N} 3-\mathrm{C} 2)$ & 116.9717 & 118.2334 & 116.9717 & 117.5751 & 116.646 & 111.52 & 111.5845 & 111.5732 & 111.4955 & 111.4631 \\
\hline$<(\mathrm{H}-\mathrm{N} 3-\mathrm{C} 2)$ & 117.5223 & 121.8932 & 120.492 & 121.2595 & 120.1653 & & & & & \\
\hline$<(\mathrm{H}-\mathrm{N} 3-\mathrm{H})$ & 119.1408 & 119.8719 & 118.2977 & 119.2068 & 118.005 & & & & & \\
\hline$<(\mathrm{N} 3-\mathrm{C} 2-\mathrm{C} 4)$ & 121.0332 & 121.0235 & 120.9648 & 120.995 & 120.9387 & 130.0397 & 130.0591 & 130.0392 & 130.022 & 130.0155 \\
\hline$<(\mathrm{N} 1-\mathrm{C} 2-\mathrm{C} 4)$ & 122.4683 & 122.4157 & 122.4869 & 122.518 & 122.5768 & 113.4705 & 113.4183 & 113.4524 & 113.5333 & 113.5411 \\
\hline$<(\mathrm{C} 2-\mathrm{C} 4-\mathrm{C} 5)$ & 117.8142 & 117.8974 & 117.9541 & 117.6982 & 117.7288 & 115.4528 & 115.526 & 115.5151 & 115.3062 & 115.2584 \\
\hline$<(\mathrm{C} 4-\mathrm{C} 5-\mathrm{N} 6)$ & 174.308 & 174.3324 & 174.5307 & 174.3675 & 174.2845 & 175.9405 & 175.9479 & 176.1684 & 176.0375 & 175.9178 \\
\hline$<(\mathrm{C} 5-\mathrm{C} 4-\mathrm{C} 7)$ & 123.7906 & 123.7474 & 123.7502 & 123.8073 & 123.7954 & 122.9664 & 123.9587 & 122.9462 & 122.9884 & 123.0184 \\
\hline$<(\mathrm{C} 2-\mathrm{C} 4-\mathrm{C} 7)$ & 118.3602 & 118.3042 & 118.2617 & 118.4554 & 118.4342 & 121.5563 & 122.5861 & 121.5128 & 121.6746 & 121.6917 \\
\hline$<(\mathrm{C} 4-\mathrm{C} 7-\mathrm{R})$ & 122.3363 & 122.1643 & 122.1825 & 122.4601 & 122.4679 & 122.5414 & 122.3631 & 122.4472 & 122.7523 & 122.8074 \\
\hline$<(\mathrm{R}-\mathrm{C} 7-\mathrm{C} 8)$ & 119.9253 & 119.9617 & 119.9652 & 120.0264 & 120.0532 & 118.1561 & 118.2169 & 118.1639 & 118.2228 & 118.2079 \\
\hline$<(\mathrm{C} 4-\mathrm{C} 7-\mathrm{C} 8)$ & 117.7369 & 117.871 & 117.8519 & 117.5115 & 117.4788 & 119.2977 & 119.4145 & 119.3843 & 119.0208 & 118.9811 \\
\hline$<(\mathrm{C} 7-\mathrm{C} 8-\mathrm{H})$ & 119.1047 & 119.122 & 119.0846 & 119.0499 & 118.9855 & 119.7813 & 119.8796 & 119.8814 & 119.803 & 119.7832 \\
\hline$<(\mathrm{H}-\mathrm{C} 8-\mathrm{C} 9)$ & 120.6845 & 120.7279 & 120.5804 & 120.6041 & 120.4862 & 119.7081 & 119.7086 & 119.6714 & 119.5531 & 119.5601 \\
\hline$<(\mathrm{C} 7-\mathrm{C} 8-\mathrm{C} 9)$ & 120.2085 & 120.1485 & 120.2513 & 120.3414 & 120.4438 & 120.5096 & 120.4115 & 120.4468 & 120.6439 & 120.6568 \\
\hline$<\left(\mathrm{C} 8-\mathrm{C} 9-\mathrm{R}^{\prime}\right)$ & 121.6657 & 121.7178 & 121.7237 & 121.7204 & 121.7044 & 123.5097 & 123.592 & 123.5564 & 123.5161 & 123.5497 \\
\hline$<\left(\mathrm{R}^{\prime}-\mathrm{C} 9-\mathrm{N} 1\right)$ & 116.3929 & 116.3853 & 116.5708 & 116.314 & 116.4877 & 117.5076 & 117.4211 & 117.4703 & 117.4512 & 117.403 \\
\hline$<(\mathrm{C} 8-\mathrm{C} 9-\mathrm{N} 1)$ & 121.9408 & 121.8967 & 121.6966 & 121.9654 & 121.7979 & 118.9804 & 118.9864 & 118.972 & 119.0314 & 119.0463 \\
\hline$<(\mathrm{C} 9-\mathrm{N} 1-\mathrm{H})$ & & & & & & 120.1788 & 120.1262 & 120.1418 & 120.2235 & 120.224 \\
\hline$<(\mathrm{C} 9-\mathrm{N} 1-\mathrm{C} 2)$ & 119.2695 & 119.3548 & 119.4355 & 119.1969 & 119.2436 & 126.1714 & 126.6437 & 126.2181 & 126.0797 & 126.0638 \\
\hline
\end{tabular}

Table 7. Some of the bond lengths (in $\AA$ ) for $\mathbf{C}$ and $\mathbf{D}$ tautomers

\begin{tabular}{l|ccccc|ccccc}
\hline \multirow{2}{*}{ Bond } & \multicolumn{3}{|c|}{ D Tautomer } & \multicolumn{5}{c}{ C Tautomer } \\
\cline { 2 - 10 } & $\mathrm{D} 1$ & $\mathrm{D} 2$ & $\mathrm{D} 3$ & $\mathrm{D} 4$ & $\mathrm{D} 5$ & $\mathrm{C} 1$ & $\mathrm{C} 2$ & $\mathrm{C} 3$ & $\mathrm{C} 4$ & $\mathrm{C} 5$ \\
\hline $\mathrm{r}(\mathrm{H})-\mathrm{r}(\mathrm{N} 1)$ & 1.01386 & 1.01388 & 1.01366 & 1.01361 & 1.01356 & & & & \\
$\mathrm{r}(\mathrm{N} 1)-\mathrm{r}(\mathrm{C} 2)$ & 1.40686 & 1.40694 & 1.40645 & 1.40635 & 1.40905 & 1.31945 & 1.31959 & 1.31926 & 1.31925 & 1.31994 \\
$\mathrm{r}(\mathrm{C} 2)-\mathrm{r}(\mathrm{O} 3)$ & 1.22646 & 1.22605 & 1.22691 & 1.22708 & 1.22725 & 1.34992 & 1.3493 & 1.35029 & 1.35038 & 1.35164 \\
r(O3)-r(H) & & & & & & 0.97061 & 0.9706 & 0.97054 & 0.97057 & 0.97013 \\
r(C2)-r(C4) & 1.46372 & 1.46373 & 1.46299 & 1.46286 & 1.4583 & 1.42195 & 1.42208 & 1.42211 & 1.42214 & 1.41823 \\
r(C4)-r(C5) & 1.4278 & 1.42751 & 1.42751 & 1.42752 & 1.42658 & 1.42514 & 1.42492 & 1.42483 & 1.42486 & 1.42424 \\
r(C5)-r(N6) & 1.16451 & 1.16453 & 1.16474 & 1.16477 & 1.16494 & 1.16683 & 1.16683 & 1.16706 & 1.16708 & 1.16692 \\
r(C4)-r(C7) & 1.39469 & 1.39474 & 1.39669 & 1.39702 & 1.39271 & 1.41387 & 1.41411 & 1.41526 & 1.41549 & 1.41229 \\
r(C7)-r(R) & 1.48742 & 1.48687 & 1.48328 & 1.4826 & 1.50717 & 1.48565 & 1.48498 & 1.48224 & 1.48158 & 1.50554 \\
r(C7)-r(C8) & 1.42374 & 1.42348 & 1.42527 & 1.42568 & 1.41803 & 1.39612 & 1.39592 & 1.39753 & 1.39791 & 1.3908 \\
r(C8)-r(H) & 1.08137 & 1.08143 & 1.08131 & 1.08132 & 1.08229 & 1.08181 & 1.08193 & 1.08184 & 1.08184 & 1.0826 \\
r(C8)-r(C9) & 1.37706 & 1.37762 & 1.37643 & 1.37612 & 1.3802 & 1.40558 & 1.40599 & 1.40456 & 1.40423 & 1.40883 \\
r(C9)-r(R') & 1.47745 & 1.47726 & 1.47781 & 1.47792 & 1.47635 & 1.48422 & 1.48358 & 1.48432 & 1.48467 & 1.48297 \\
r(C9)-r(N1) & 1.3663 & 1.36598 & 1.36645 & 1.36661 & 1.36598 & 1.34696 & 1.34676 & 1.34746 & 1.34754 & 1.34738 \\
\hline
\end{tabular}

This comparison has been made on the basis of the energy level of tautomers (Table 4) as well as the single and double bond lengths in the pyrimidine ring (Fig. $1 \&$ Table 5-8). On the basis of this comparison, a more effective resonance seems to occur in the pyrimidine ring of tautomers $\mathbf{B}$ and $\mathbf{D}$ relative to $\mathbf{A}$ and $\mathbf{C}$. The comparison between the energy of tautomers shows that $\mathbf{B}$ tautomers are much more stable than A tautomers. In the case of $\mathbf{D}$ and $\mathbf{C}$ tautomers, the energy differences decrease compared to $\mathbf{B}$ and $\mathbf{A}$ tautomers, but are still noticeable. The pyrimidine ring in $\mathbf{D}$ tautomers shows more effective resonance.

The equilibrium constants between $\mathbf{B}$ and $\mathbf{A}$ and also $\mathbf{D}$ and $\mathbf{C}$ using $\Delta G$ could be calculated using:

$$
K=\exp \left(-\frac{\Delta G}{R T}\right)
$$

The equilibrium constants related to $\mathbf{B} \rightleftharpoons \mathbf{A}$ are about $10^{-12}$, showing that tautomers $\mathbf{B}$ are dominant. Although the equilibrium constants related to $\mathbf{D} \rightleftharpoons \mathbf{C}$ have increased to about $10^{-2}-10^{-1}$, the tautomers $\mathbf{D}$ are still predominant (Table 
Table 8. Some of the dihedral angels (in degrees) for $\mathbf{C}$ and $\mathbf{D}$ tautomers

\begin{tabular}{|c|c|c|c|c|c|c|c|c|c|c|}
\hline \multirow{2}{*}{ Angel } & \multicolumn{5}{|c|}{ D Tautomer } & \multicolumn{5}{|c|}{ C Tautomer } \\
\hline & D1 & D2 & D3 & D4 & D5 & $\mathrm{C} 1$ & $\mathrm{C} 2$ & $\mathrm{C} 3$ & $\mathrm{C} 4$ & $\mathrm{C} 5$ \\
\hline$<(\mathrm{H}-\mathrm{N} 1-\mathrm{C} 2)$ & 113.6556 & 113.6503 & 113.7127 & 113.7245 & 113.566 & & & & & \\
\hline$<(\mathrm{N} 1-\mathrm{C} 2-\mathrm{O} 3)$ & 119.6569 & 119.706 & 119.6154 & 119.5915 & 119.5935 & 114.9166 & 114.8992 & 114.885 & 114.8768 & 114.9272 \\
\hline$<(\mathrm{C} 2-\mathrm{O} 3-\mathrm{H})$ & & & & & & 110.7803 & 110.8928 & 110.7825 & 110.7794 & 110.7346 \\
\hline$<(\mathrm{O} 3-\mathrm{C} 2-\mathrm{C} 4)$ & 126.5589 & 126.5326 & 126.5324 & 126.5447 & 127.0979 & 121.6004 & 121.6343 & 121.5708 & 121.5665 & 121.808 \\
\hline$<(\mathrm{N} 1-\mathrm{C} 2-\mathrm{C} 4)$ & 113.7838 & 113.7608 & 113.852 & 113.8637 & 113.3059 & 123.4821 & 123.4656 & 123.543 & 123.5557 & 123.2648 \\
\hline$<(\mathrm{C} 2-\mathrm{C} 4-\mathrm{C} 5)$ & 115.7131 & 115.7609 & 115.521 & 115.4651 & 117.511 & 117.641 & 117.6807 & 117.5123 & 117.4362 & 119.1825 \\
\hline$<(\mathrm{C} 4-\mathrm{C} 5-\mathrm{N} 6)$ & 179.4065 & 179.608 & 179.4991 & 179.4113 & 177.4351 & 173.4512 & 173.6208 & 173.5576 & 173.4063 & 176.4447 \\
\hline$<(\mathrm{C} 5-\mathrm{C} 4-\mathrm{C} 7)$ & 122.918 & 122.9001 & 122.9584 & 122.9994 & 120.7817 & 124.308 & 124.2892 & 124.3016 & 124.3453 & 122.6108 \\
\hline$<(\mathrm{C} 2-\mathrm{C} 4-\mathrm{C} 7)$ & 121.3468 & 121.3175 & 121.4937 & 121.5071 & 121.7062 & 118.0037 & 117.98 & 118.133 & 118.1605 & 118.2055 \\
\hline$<(\mathrm{C} 4-\mathrm{C} 7-\mathrm{R})$ & 122.7033 & 122.6078 & 122.9245 & 122.9974 & 120.7624 & 122.3307 & 122.2969 & 122.4963 & 122.5487 & 120.6748 \\
\hline$<(\mathrm{R}-\mathrm{C} 7-\mathrm{C} 8)$ & 118.0057 & 118.0304 & 118.1019 & 118.0694 & 119.6803 & 120.2979 & 120.2786 & 120.3957 & 120.4005 & 121.7994 \\
\hline$<(\mathrm{C} 4-\mathrm{C} 7-\mathrm{C} 8)$ & 119.287 & 119.3575 & 118.9697 & 118.9297 & 119.555 & 117.3704 & 117.4229 & 117.1054 & 117.0494 & 117.5255 \\
\hline$<(C 7-C 8-H)$ & 119.5417 & 119.6418 & 119.5713 & 119.5456 & 119.9123 & 118.6783 & 118.8377 & 118.7349 & 118.7428 & 118.9259 \\
\hline$<(\mathrm{H}-\mathrm{C} 8-\mathrm{C} 9)$ & 119.4694 & 119.4296 & 119.2972 & 119.3075 & 119.3639 & 120.7782 & 120.669 & 120.6091 & 120.5803 & 120.6514 \\
\hline$<(\mathrm{C} 7-\mathrm{C} 8-\mathrm{C} 9)$ & 120.988 & 120.9282 & 121.1315 & 121.147 & 120.6773 & 120.536 & 120.487 & 120.6491 & 120.6696 & 120.4168 \\
\hline$<\left(\mathrm{C} 8-\mathrm{C} 9-\mathrm{R}^{\prime}\right)$ & 123.8848 & 123.939 & 123.8794 & 123.9217 & 124.0164 & 122.3342 & 122.2766 & 122.2417 & 122.2343 & 122.3949 \\
\hline$<\left(\mathrm{R}^{\prime}-\mathrm{C} 9-\mathrm{N} 1\right)$ & 117.7918 & 117.7413 & 117.7286 & 117.6756 & 117.7448 & 116.2481 & 116.2901 & 116.2221 & 116.1978 & 116.3084 \\
\hline$<(\mathrm{C} 8-\mathrm{C} 9-\mathrm{N} 1)$ & 118.322 & 118.3189 & 118.3914 & 118.4022 & 118.2319 & 121.4173 & 121.4324 & 121.5357 & 121.5676 & 121.2939 \\
\hline$<(\mathrm{C} 9-\mathrm{N} 1-\mathrm{H})$ & 119.9854 & 119.9423 & 120.038 & 120.0372 & 119.771 & & & & & \\
\hline$<(\mathrm{C} 9-\mathrm{N} 1-\mathrm{C} 2)$ & 126.2604 & 126.3043 & 126.1451 & 126.1319 & 126.5202 & 119.182 & 119.2037 & 119.0277 & 118.9902 & 119.2878 \\
\hline
\end{tabular}

Table 9. Equilibrium constants related to $\mathbf{B} \rightleftharpoons \mathbf{A}$ and $\mathbf{D} \rightleftharpoons \mathbf{C}$

\begin{tabular}{lcc}
\hline $\mathrm{R}$ & $\begin{array}{c}\text { Equilibrium constants }(\mathrm{K}) \\
\text { for } \mathbf{B} \rightleftharpoons \mathbf{A}\end{array}$ & $\begin{array}{c}\text { Equilibrium constants }(\mathrm{K}) \\
\text { for } \mathbf{D} \rightleftharpoons \mathbf{C}\end{array}$ \\
\hline $\mathrm{C}_{6} \mathrm{H}_{5}$ & $1.26 \times 10^{-12}$ & $1.19 \times 10^{-1}$ \\
$4-\mathrm{BrC}_{6} \mathrm{H}_{4}$ & $8.85 \times 10^{-13}$ & - \\
$4-\mathrm{ClC}_{6} \mathrm{H}_{4}$ & $1.27 \times 10^{-13}$ & $1.80 \times 10^{-2}$ \\
$4-\mathrm{HOC}_{6} \mathrm{H}_{4}$ & $1.26 \times 10^{-12}$ & $2.28 \times 10^{-2}$ \\
$4-\mathrm{CH}_{3} \mathrm{OC}_{6} \mathrm{H}_{4}$ & $1.64 \times 10^{-13}$ & $2.66 \times 10^{-2}$ \\
$\mathrm{CH}_{3}$ & - & $1.50 \times 10^{-2}$ \\
\hline
\end{tabular}

9).

A plausible mechanism for the formation of compounds B1-B5 is depicted in Scheme 4. We propose that the reactions occur via initial formation of the dicyano olefin I through the Knoevenagel condensation between aryl aldehydes and malononitrile, which then reacts with intermediate II produced from the reaction of 3,4-dimethoxyacetophenone with ammonium acetate, to give the intermediate III, which subsequently undergoes the cyclization followed by air oxidation to afford the desired compounds

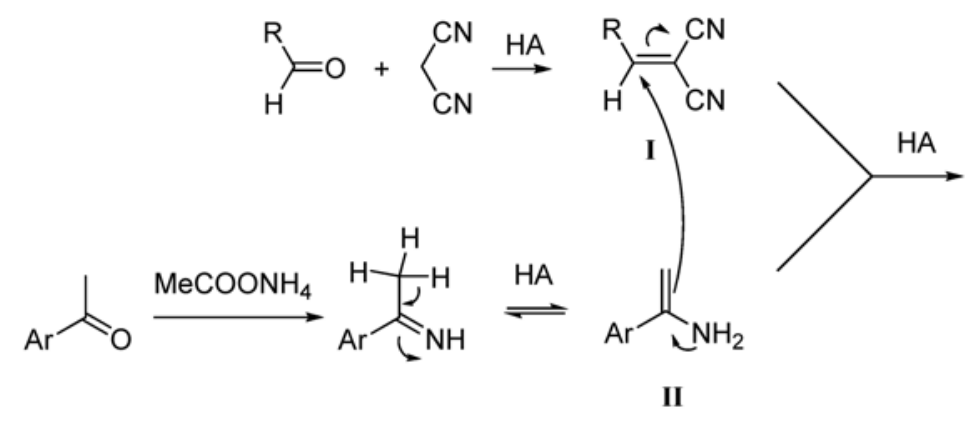

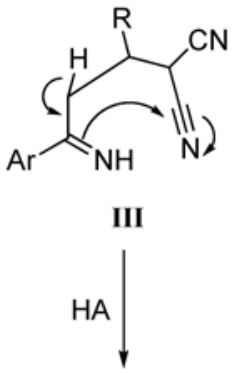<smiles>[R]c1cc([Al])nc(N)c1C#N</smiles><smiles>[R]c1cc([Al])[nH]c(=N)c1C#N</smiles><smiles>[R]C1C=C([Te])NC(=N)C1C#N</smiles>

IV

Scheme 4. Plausible mechanism for the formation of 2-amino-4-aryl-3-cyano-6-(3,4-dimethoxyphenyl)pyridines in the presence of $\left[\mathrm{HO}_{3} \mathrm{~S}\left(\mathrm{CH}_{2}\right)_{4} \mathrm{MIM}\right]\left[\mathrm{HSO}_{4}\right] \equiv \mathrm{HA}$ as catalyst. 
B1-B5 via the intermediate IV and V. The catalyst $\left[\mathrm{HO}_{3} \mathrm{~S}\left(\mathrm{CH}_{2}\right)_{4} \mathrm{MIM}\right]\left[\mathrm{HSO}_{4}\right] \mathrm{HA}$ facilitates the formation of the intermediates I-IV. Under these conditions, attempts to isolate the intermediates $\mathbf{I}-\mathbf{V}$ by careful monitoring of reactions failed. Compounds D1-D5 can also be formed by a similar route.

\section{Conclusions}

A series of 2-amino-4-aryl-3-cyano-6-(3,4-dimethoxyphenyl)pyridines and 4-aryl(or alkyl)-3-cyano-6-(3,4-dimethoxyphenyl)-2(1H)-pyridinones has been prepared through a facile one-pot solvent-free multicomponent reaction using $\left[\mathrm{HO}_{3} \mathrm{~S}\left(\mathrm{CH}_{2}\right)_{4} \mathrm{MIM}\right]\left[\mathrm{HSO}_{4}\right]$ as the catalyst. The experimental results are consistent with the theoretical calculation regarding the formation of more stable tautomers.

Acknowledgments. The authors are thankful to Islamic Azad University, Mashhad Branch for financial support.

\section{References}

1. Konda, S. G.; Khedkar, V. T.; Dawane, B. S. J. Chem. Pharm. Res. 2010, 2,187

2. Mungra, D. C.; Patel, M. P.; Patel, R. G. Arkivoc 2009, 64.

3. Vyas, D. H.; Tala, S. D.; Akbari, J. D.; Dhaduk, M. F.; Joshi, K. A.; Joshi, H. S. Ind. J. Chem. B-Org. Chem. Incl. Med. Chem. 2009, 48, 833.

4. Gholap, A. R.; Toti, K. S.; Shirazi, F.; Kumari, R.; Bhat, M. K.; Deshpande, M. V.; Srinivasan, K. V. Bioorg. Med. Chem. 2007, 15,6705 .

5. Bekhit, A. A.; Baraka, A. M. Eur. J. Med. Chem. 2005, 40, 1405.

6. Murata, T.; Shimada, M.; Sakakibara, S.; Yoshino, T.; Kadono, H.; Masuda, T.; Shimazaki, M.; Shintani, T.; Fuchikami, K.; Sakai, K.; Inbe, H.; Takeshita, K.; Niki, T.; Umeda, M.; Bacon, K. B.; Ziegelbauer, K. B.; Lowinger, T. B. Bioorg. Med. Chem. Let. 2003, 13, 913 .
7. Abadi, A. H.; Al-Khamees, H. A. Arch. Pharm. 1998, 331, 319.

8. Thompson, P. E.; Manganiello, V.; Degerman, E. Cur. Top. Med. Chem. 2007, 7, 421.

9. Abadi, A.; Al-Deeb, O.; Al-Afify, A.; El-Kashef, H. Farmaco 1999, 54, 195.

10. Abadi, A. H.; Abouel-Ella, D. A.; Lehmann, J.; Tinsley, H. N.; Gary, B. D.; Piazza, G. A.; Abdel-Fattah, M. A. O. Eur. J. Med. Chem. 2010, 45, 90.

11. Kambe, S.; Saito, K.; Sakurai, A.; Midorikawa, H. Synthesis 1980, 366.

12. Paul, S.; Gupta, R.; Loupy, A. J. Chem. Res. S 1998, 330.

13. Shaker, R. M.; AbdelLatif, F. F. J. Chem. Res. S 1997, 294.

14. Shi, F.; Tu, S. J.; Fang, F.; Li, T. J. Arkivoc 2005, 137.

15. Litvinov, V. P.; Yakunin, Y. Y.; Dyachenko, V. D. Chem. Hetero. Comp. 2001, 37, 37.

16. Heravi, M. M.; Beheshtia, Y. S.; Khorshidi, M.; Baghernejad, B.; Bamoharam, F. F. Chin. J. Chem. 2009, 27, 569.

17. Alberola, A.; Calvo, L. A.; Ortega, A. G.; Ruiz, M. C. S.; Yustos, P.; Granda, S. G.; Garcia-Rodriguez, E. J. Org. Chem. 1999, 64, 9493.

18. Litvinov, V. P. Rus. Chem. Rev. 2006, 75, 577.

19. Davoodnia, A.; Bakavoli, M.; Barakouhi, Gh.; Tavakoli-Hoseini, N. Chin. Chem. Lett. 2007, 18, 1483.

20. Davoodnia, A.; Roshani, M.; Malaeke, S. H.; Bakavoli, M. Chin. Chem. Lett. 2008, 19, 525.

21. Davoodnia, A.; Heravi, M. M.; Rezaei-Daghigh, L.; TavakoliHoseini, N. Monatsh. Chem. 2009, 140, 1499.

22. Davoodnia, A.; Bakavoli, M.; Moloudi, R.; Khashi, M.; TavakoliHoseini, N. Monatsh. Chem. 2010, 141, 867.

23. Davoodnia, A.; Heravi, M. M.; Rezaei-Daghigh, L.; TavakoliHoseini, N. Chin. J. Chem. 2010, 28, 429.

24. Davoodnia, A.; Heravi, M. M.; Safavi-Rad, Z.; Tavakoli-Hoseini, N. Synth. Commun. 2010, 40, 2588.

25. Tavakoli-Hoseini, N.; Davoodnia, A. Asian J. Chem. 2010, 22, 7197.

26. Davoodnia, A.; Allameh, S.; Fakhari, A. R.; Tavakoli-Hoseini, N. Chin. Chem. Lett. 2010, 21, 550.

27. Davoodnia, A.; Bakavoli, M.; Moloudi, R.; Khashi, M.; TavakoliHoseini, N. Chin. Chem. Lett. 2010, 21, 1.

28. Wang, W. J.; Shao, L. L.; Cheng, W. P.; Yang, J. G.; He, M. Y. Catal. Commun. 2008, 9, 337. 